analysiert and gefunden, daß die Reaktion dem Dracoresin und dem Dracoresenharz zukommt, nicht dagegen dem Dracoalban und dem Dracoresinotannol.

Kino liefert nach Hlasiwetz bei der Kalischmelze etwa 9\% Phloroglucin, worans sich das Auftreten der Reaktion erklärt.

Dagegen liefert Myrrha bei der Kalischmelze Brenzkatechin and Protokatechusänre, zwei Körper, die beide die Reaktion nicht gaben. Wir haben das Harz dann nach den Angaben von Tucholka (Ueber die Bisabol-Myrrha. Diss. Zïrich 1897) und Köhler (Arch. d. $\mathrm{Ph} .1890$, S. 291) analysiert. Es worden dargestellt das atherische Oel, eine freie Harzsäure, zwei gebundene Harzsäuren, von denen die eine ein unlösliches, die andere ein losliches Bleisalz lieferte and ein Bitterstoff. Von diesen Körpern gab das ätherische Oel und die mit Bleiacetat nicht fällbare Sänre mit Vanillin-Salzsăure Rotfärbung.

Wir hoffen diesen mehr orientierenden Mitteilungen bald weitere folgen za lassen.

\title{
Ueber die Gegenwart von Lecithin im Weine.
} (Bemerkungen zu der Abhandlung von G. Ortlieb und
J. Weirich.)*) Von Dr. A. Rosenstiehl.

(Eingegangen den 21. VI. 1904.)

Die Entdeckung von organisch gebundenem Phosphor im Weine erhalt Wichtigkeit durch den Nährwert, welcher demselben in Gestalt von Lecithin zugeschrieben wird. Diese Entdeckung ist das Verdienst von G. Ortlieb und J. Weirich. Sie lehrt uns, daß die krăftigende Wirkung des Weines nicht allein dem Alkohol zuzuschreiben ist.

Leider ist aber die Gegenwart des Lecithins experimentell bisher nur für einen einzigen Wein festgestellt. Es ist dies ein Wein, der aus trockenen Trauben der Insel Thyra bereitet wurde. Er enthall 15\% Alkohol und einen Ueberschuß von Traubenzucker; derselbe soll als Wein für Kranke Verwendang finden. Es handelt sich also zunächst nur um einen Einzelfall. Bei der Bestimmung des organisch gebundenen Phosphors ist den Verfassern ein negatives Resultat aufgefallen, woraus sie wichtige, aber auch unberechtigte Schlüsse ziehen. Als sie nämlich eine Probe dieses Weines bei $80^{\circ} \mathrm{C}$. eindampften,

*) Dieses Archiv 242, 138. 
konnten sie in dem Rückstande keinen organisch gebundenen Phosphor mehr finden; in Proben, welche bei $50^{\circ} \mathrm{C}$. eingetrocknet wurden, erhielten sie dagegen sehr übereinstimmende und befriedigende Resultate. Den Unterschied schreiben die Verfasser dem höheren Wărmegrade zo, und schließen daraus, daß wenig liber $50^{\circ} \mathrm{C}$. das Lecithin vollständig zerstört wird. Dabei aber vergessen sie, daß sie im ersten Falle den Wein mit kohlensaurem Kalk neutralisiert hatten, was bei den anderen Analysen nicht der Fall war. Sie hatten zwei Bedingungen geändert, und nur der einen Rechnung getragen. Die Grundlage dieses ersten Schlusses ist also nicht experimentell festgelegt und demnach hinf ăllig.

Trotz dieses mangelhaften Ausgangspunktes, folgern die Verfasser, daß das Pasteurisieren der Weine und das Erhitzen der Moste - Rosenstiehl'sches Verfahren - sicher den Hauptbestandteil des Weines, das Lecithin, zerstören, das sich schon wenig über $50^{\circ} \mathrm{C}$. zersetzt. Infolgedessen warnen sie vor diesen Manipulationen im Interesse der physiologischen Wirkung des Weines und verurteilen ohne weiteres die Pasteurisation und die Sterilisation der Moste. Jedoch erkennen sie an, daß beide sicher gute Methoden sind, um die schädlichen Pilze, Ursachen der bekanntesten Weinkrankheiten zu zerstören oder an der Weiterentwickelung zu verhindern. Sie scheinen rationell und angezeigt . . . aber sie hinterlassen ein Gemisch, das, so sehr es auch dem Gaumen des Konsumenten munden mag ... den Namen Wein, im rein wissenschaftlichen Sinne nicht mehr verdient.

Alle die wichtigen Schlüsse sind aus einer verfehlten Analyse und der Hypothese entstanden: Alle Weine enthalten wehr oder weniger Lecithin! Denn die Verfasser haben nicht etwa einen Wein vor oder nach dem Pasteurisieren analysiert, ebensowenig haben sie einen aus sterileun Moste erhaltenen Wein gegen seinen Kontrollwein antersucht. Alle ihre Folgerungen beruhen lediglich auf der Phosphorbestimmung eines aus trockenen Trauben bereiteten Weines! Zwischen der zu Grunde gelegten Tatsache und den schweren Folgen besteht somit kein Verhältnis.

Es erscheint nicht angezeigt, die angeschuldigten Methoden weiter zu verteidigen. Sie haben ihre Proben abgelegt, und die Dienste, die sie leisten, bewahren ihren Wert. Es soll hier nur dasjenige besprochen werden, was sich auf unsere jetzigen Kenntnisse t̄ber die Einwirkung der Warme auf Lecithin bezieht.

Bekanntlich ist das letztere nur dann gegen Wärme empfindlich, wenn es in konzentriertem Zustande sich befindet, sowie es beim Eindampfen seiner Lösungen oder Emulsionen erhalten wird. Deshalb 
wird bei der Analyse der niedrigste anwendbare Temperaturgrad innegehalten and $50^{\circ} \mathrm{C}$. nicht überschritten. Ist aber Lecithin in ver: dünntem Zustande - und in einem solchen nur kann es im Weine erwartet werden - dann ist es merkwürdig beständig.

Für den Wein liegen zwar keine Zahlen vor. Es ist von den Verfassern anch nicht direkt ermittelt worden, ob die Weine uberhanpt Lecithin enthalten; es ist daher zu wünschen, daß sie diese Lücke in ihrer Arbeit ausfullen werden.

Für die Milch aber liegen genaue Angaben vor. (Bordas und Raczkowski CR. T. CXXXVI, S. 56.)

Wird Milch auf $95^{\circ} \mathrm{C}$. im Wasserbade erhitzt, so beträgt der Verlust an Lecithin in einer halben Stunde $12 \%$. Die Milch enthält im Liter $0,250 \mathrm{~g}$ bis $0,365 \mathrm{~g}$ Lecithin. Im Thyrawein haben G. Ortlieb und J. Weirich $0,0352 \mathrm{~g}$ Lecithin gefunden. Also ganz vergleichbare Zahlen. Beim Pasteurisieren oder dem Sterilisieren der Moste werden so hohe Temperaturen nie erreicht, und auch die Dauer der Erwärmung beschränkt sich auf wenige Minuten. Demnach ist bei der Einwirkung der Wärme auf Weine oder Moste keine der Bedingungen erfüllt, bei der die Zerstörung des Lecithins auch nur $12 \%$ erreichen könnte. Wäre ubrigens das Lecithin so empfindlich, wie die Verfasser es schildern, so müßte dasselbe für unsere Nahrungsmittel auch der Fall sein. Denn Eigelb, grüne und trockene Gemüse, Samen, Fritchte and Fleisch verdanken der Gegenwart von Lecithin einen Teil ihres Nahrwertes. Die Verfasser wären also gleichberechtigt vor dem Kochen dieser Nahrungsmittel zu warnen, um so mebr, da die Temperaturen und besonders die Zeiten bedeutend großer sind als beim Pasteurisieren. Diese einfache Folgerung zeigt nun das Unhaltbare der aufgestellten Schltisse.

Diese Besprechung kann daher in folgender Weise zusammengefaßt werden:

1. Die Gegenwart von Lecithin in den verschiedenen Weinen ist durch die Verfasser nicht bewiesen worden.

2. Ebensowenig dessen Zerstorrang in Weinen durch die Pasteurisation, noch in Mosten durch deren Erhitzen.

3. Es geht im Gegenteil aus den bekannten Tatsachen hervor, daB beim Pasteurisieren oder Sterilisieren der Moste, keine der Bedingungen erfüllt ist, in denen das Lecithin zerstört werden könnte. 